\title{
Management of grade 3 acute dermatitis with moist desquamation after adjuvant chest wall radiotherapy: a case report
}

\author{
Durim Delishaj ${ }^{1}$, Romerai D'amico ${ }^{1}$, Daniela Corvi ${ }^{1}$, Giuseppe De Nobili ${ }^{1}$, Alessandro Alghisi ${ }^{1}$, Francesco \\ Colangelo ${ }^{2}$, Alessandra Cocchi ${ }^{1}$, Fausto Declich ${ }^{3}$, Carlo Pietro Soatti ${ }^{1}$ \\ ${ }^{1}$ Department of Radiation Oncology, Alessandro Manzoni Hospital, Lecco, Italy \\ ${ }^{2}$ Department of Radiation Oncology, University of Milano Bicocca, Milan, Italy \\ ${ }^{3}$ Medical Physics Unit, Alessandro Manzoni Hospital, Lecco, Italy
}

Received: November 21, 2020

Accepted: November 30, 2020

Correspondence:

Durim Delishaj

Department of Radiation Oncology, Alessandro Manzoni Hospital, Street Dell'Eremo 9/11, Lecco-23900,

Lombardy, Italy

Tel: $+39-341253540$

Fax: $+39-341489710$

E-mail: delishaj@hotmail.com

ORCID:

https://orcid.org/0000-0002-1782-0852
We reported a successful case management of G3 skin acute dermatitis in a 32-year-old woman affected by locally advanced breast cancer underwent adjuvant chest wall irradiation. Skin acute toxicity with dry desquamation areas was treated daily with advanced dressing using physiological solution, oxygen therapy and applying hyaluronic acid gauze. At the end of radiotherapy treatment, G3 skin acute dermatitis with moist desquamation was observed, so the patient continued advanced wound dressing shifted to twice weekly with physiological solution, oxygen therapy and applying hydrocolloid dressing. The patient completed radiotherapy treatment without interruption and one month after treatment acute skin toxicity was resolved with pain relief. We suggest that advanced dressing with trained nursing staff is essential in this sub-set of patients due to guaranteed continuation of radiotherapy treatment, indispensable to ensure patient cure.

Keywords: Breast cancer, Radiotherapy, Dermatitis, Toxicity, Moist desquamation, Wound dressing

\section{Introduction}

Breast cancer is the most common female malignancy [1]. In the majority of patients, adjuvant radiation therapy, after conserving breast surgery or post-mastectomy, is indicate to improve locoregional control rate and overall survival [2]. External beam radiotherapy (EBRT) or high-dose rate brachytherapy are used for chest wall irradiation after mastectomy, re-irradiation or skin recurrences in patients with breast cancer. It is known that conventional radio-therapy treatment must be delivered daily and its interruption for long interval of time can influence efficacy and consequently locoregional control [3].

Adjuvant radiation therapy for breast cancer is generally well tolerated, however, acute skin toxicity is a common side effect that impacts quality of life especially in patients receiving either adjuvant chemotherapy [4]. Patients with breast cancer can develop severe acute radiation-induced skin reactions because their skin receives a relatively high dose as the tumor is close to the skin $[4,5]$.

Most common symptoms of radiation induced dermatitis include irritation, pain, itching, peeling, and moist desquamation $[5,6]$. Moist desquamation (MD) occurs typically after a cumulative doses of 30 Gy as a result of destruction, sloughing of dermal layers and is characterized by serous fluid drainage and painful. MD often begins as small patches in skin folds and can progress to involve larger, confluent areas of irradiated skin.

Copyright (C) 2020 The Korean Society for Radiation Oncology

This is an Open Access article distributed under the terms of the Creative Commons Attribution Non-Commercial License (http://creativecommons.org/licenses/by-nc/4.0/) which permits unrestricted non-commercial use, distribution, and reproduction in any medium, provided the original work is properly cited. 
The risk of developing MD is highly variable and depending on radiation target as well as multiple patient and treatment factors. Typically the incidence rate are higher after post-mastectomy radiation therapy (with rates about 71\%) compared to breast conservation (range, $11 \%$ to $47 \%$ ) [7].

According to the Radiation Therapy Oncology Group (RTOG) and the Common Terminology Criteria for Adverse Events (CTCAE) scale, MD is classified as Grade 3 (G3) skin toxicity $[8,9]$. Some trials reported benefits using advanced dressing with significant lower levels of pain, burning, edema and management of MD in patients with higher grade acute skin toxicity [10-19].

We reported a successful case management of G3 skin acute dermatitis in a 32-year-old women affected by locally advanced breast cancer underwent adjuvant chest wall irradiation.

\section{Case Report}

In our center, about 300 patients with breast cancer receive adjuvant radiotherapy after surgery. We reported the case of a 32-yearold woman affected by locally advanced breast cancer. At the beginning of 2019 at self-examination, the patient noticed a lump on right breast with deformation of the nipple profile, for this reasons underwent clinical and imaging examination. The patient signed informed consent before starting radiotherapy treatment.

A breast magnetic resonance imaging (MRI) showed multifocal alterations of heteroplastic significance in lower quadrants of the right breast, characterized by increased gadolinium enhancement. Ultrasound guided fine-needle aspiration biopsy (FNAB) was positive for infiltrating ductal carcinoma. A computed tomography (CT) total body and bone scan had negative finding for distant metastasis. So, after multidisciplinary board evaluation, the patient underwent modified radical mastectomy with axillary lymph node dissection.

The histopathological examination confirmed multifocal infiltrating ductal carcinoma, G2, presence of perineural infiltration
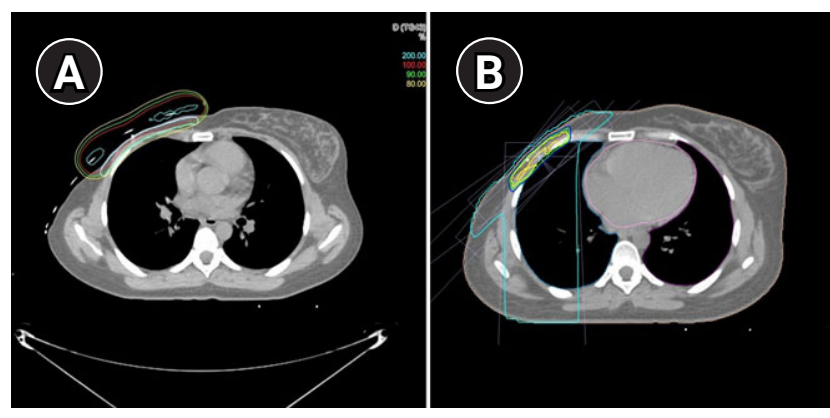

Fig. 1. Radiotherapy treatment planning using brachytherapy technique $(A)$ and external beam radiation therapy technique (B). and focal endolymphatic invasion. The neoplasm infiltrated the striated muscle on the deep plane and coincided with margin for a maximum linear extension $1.3 \mathrm{~mm}$, respectively. Eight axillary lymph nodes examined resulted free from cancer cells. The TNM histological stage resulted $\mathrm{pT} 2(\mathrm{~m}) \mathrm{NOMO}, \mathrm{G} 2, \mathrm{R} 1$, estrogen receptor (ER) positive, progesterone receptor (PgR) positive, human epidermal growth factor receptor 2 (HER2) negative, and Ki-67 (40\%).

After multidisciplinary breast board discussion and according to international breast cancer guidelines the patient was candidate to chemotherapy regimen with epirubicin-cyclophosphamide for four cycle and sequentially weekly paclitaxel for 12 cycle, aromatase inhibitors for 5 years and adjuvant chest wall radiotherapy.

From May 11, 2020 to June 12, 2020, the patient underwent chest wall irradiation with conventional fractionation at the dose of $46 \mathrm{~Gy}$ in 23 fractions using high-dose rate brachytherapy technique (Fig. 1A). A sequential boost of $14 \mathrm{~Gy}$ in 7 fractions was delivered using photon EBRT technique to positive margin, identified by the metal clips positioned during the surgery (Fig. 1B). Radiotherapy planning treatment was evaluated by radiation oncologist and all dose constraints for organs-at-risk were respected.

At the dose of $34 \mathrm{~Gy}$, erythema G2 was observed which require to shift of local therapy from emollient cream to hyaluronic acid cream, commonly used for skin repair due to radiation damage. In addition, the patient reported mild pain controlled with non-steroidal anti-inflammatory drugs (NSAIDs).

At the dose of $46 \mathrm{~Gy}, \mathrm{G} 2$ acute toxicity evolved with comparison of dry desquamation areas, so the patient needed advanced daily dressing with physiological solution, oxygen therapy and applying hyaluronic acid gauze with clinical benefit.

The patient completed radiotherapy treatment at the prescribed dose without interruption it. At the end of radiotherapy treatment skin acute toxicity evolved to G3 dermatitis with onset of MD at the irradiation area (Fig. 2A). For this reason, the patient continued advanced wound dressing shifted to twice weekly with physiological solution, oxygen therapy and applying hydrocolloid dressing.

Hydrocolloid dressing contains calcium alginate, which creates the ideal moist environment for the healing process of skin lesions by increasing perspiration based on the amount of exudate and keeping the lesion at constant temperature and humidity. One month after treatment $\mathrm{G} 3$ toxicity was resolved with pain relief and only topical emollient cream was applied (Fig. 2B).

\section{Discussion}

Adjuvant radiotherapy for breast cancer is well tolerated and radiation dermatitis is one of the most common side effects [1-3]. Typically, the incidence rate of radio-induced dermatitis is higher after 


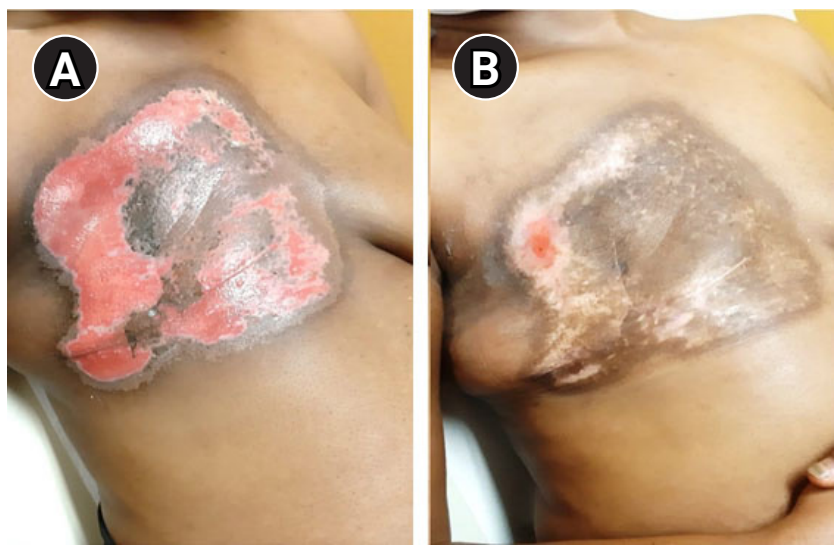

Fig. 2. Skin acute dermatitis (grade 3 ) during radiotherapy treatment (A) and resolution with advanced nursing dressing one month after treatment (B).

post-mastectomy radiation compared to breast conservation and usually consist in low-grade toxicity [4-6]. The use of new radiation techniques such as breast intensity-modulated radiation therapy seems to reduce the incidence of dermatitis in patients with breast cancer underwent adjuvant radiotherapy [16].

Emollient cream are widely used for the prevention and treatment of low grade acute dermatitis. Various studies analyzed the role topical therapy for the prevention and treatment of radiation induced dermatitis comparing different emollient cream, but failed to demonstrate the superiority of one over another [10-13]. Zhang et al. [12] published a meta-analysis of 20 reports of clinical trials using topical agents for prevention and treatment of radiodermatitis. In this analyses were included 3,098 patients and authors concluded that current topical agents do not prevent or treat radiation dermatitis effectively.

In the study of Nasser et al. [13], topical vitamin D ointment was not superior to aqua cream for prevention of radiation-induced dermatitis in women treated with adjuvant radiation for breast cancer. With the exception of corticosteroids, many different pharmacological topical treatments failed to consistently decrease skin reaction severity above standard care in breast cancer and head and neck cancer patients.

Bostrom et al. [15] in a double-blind randomized study showed that mometasone furoate, a potent corticosteroid cream, significantly reduces acute radiation dermatitis. Similar results were reported by other randomized clinical trials using mometasone furoate for the prevention of radiation induced dermatitis $[16,17]$.

A randomized double-blinded phase 3 trial due clarify the clinical benefit of topical steroid for radiation dermatitis induced by high-dose irradiation with chemotherapy is ongoing (the study protocol of J-SUPPORT 1602) and the results will be soon available.

There are not a lot of data in literature regarding the manage- ment of G2/3 dermatitis after radiotherapy treatment and empirical treatment with antibiotics and corticosteroids is recommended, but often without immediate benefit and radiotherapy interruption is unavoidable due to prevent aggravation and complication of radiation induced dermatitis.

We reported a successful case management of G3 dermatitis using advanced dressing in a woman with breast cancer underwent chest wall adjuvant radiotherapy. The patient completed radiotherapy treatment without interruption despite comparison of G2/G3 dermatitis during radiotherapy treatment.

Some studies reported a clinical benefit using advanced dressing for $\mathrm{G} 2 / 3$ or highs radiation induced dermatitis [18-20]. In a phase I study, the use of topical epigallocatechin-3-gallate in patients with breast cancer receiving adjuvant radiotherapy appear to be effective in treating radiation dermatitis in this preliminary investigation [19]. Moreover, in a randomized intra-patient controlled clinical trial, Yan et al. [20] showed that Mepitel Film was superior to Biafine cream in reducing the severity of acute G3 radiation-induced skin reactions and moist desquamation incidence in head and neck patients.

In conclusion, we suggest advanced dressing with trained nursing staff for the management of G2/G3 radiation-induced dermatitis due to prevent aggravation of dermatitis and infectious complications. A trained nursing staff for an adequate management of G2/G3 radiation induced dermatitis is essential in this sub-set of patients due to guaranteed continuation of radiotherapy treatment, indispensable to ensure patient cure.

\section{Conflict of Interest}

No potential conflict of interest relevant to this article was reported.

\section{References}

1. Veronesi U, Cascinelli N, Mariani $L$, et al. Twenty-year follow-up of a randomized study comparing breast-conserving surgery with radical mastectomy for early breast cancer. $\mathrm{N}$ Engl J Med 2002;347:1227-32.

2. Fisher B, Anderson S, Bryant J, et al. Twenty-year follow-up of a randomized trial comparing total mastectomy, lumpectomy, and lumpectomy plus irradiation for the treatment of invasive breast cancer. N Engl J Med 2002;347:1233-41.

3. Early Breast Cancer Trialists' Collaborative Group (EBCTCG). Effect of radiotherapy after breast-conserving surgery on 10-year recurrence and 15-year breast cancer death: meta-analysis of individual patient data for 10,801 women in 17 randomised trials. 
Lancet $2011 ; 378: 1707-16$.

4. Drost L, Li N, Vesprini D, et al. Prospective study of breast radiation dermatitis. Clin Breast Cancer 2018;18:e789-e795.

5. Butcher K, Williamson K. Management of erythema and skin preservation; advice for patients receiving radical radiotherapy to the breast: a systematic literature review. J Radiother Pract 2012; $11: 44-54$.

6. Wong RK, Bensadoun RJ, Boers-Doets CB, et al. Clinical practice guidelines for the prevention and treatment of acute and late radiation reactions from the MASCC Skin Toxicity Study Group. Support Care Cancer 2013;21:2933-48.

7. Cox JD, Stetz J, Pajak TF. Toxicity criteria of the Radiation Therapy Oncology Group (RTOG) and the European Organization for Research and Treatment of Cancer (EORTC). Int J Radiat Oncol Biol Phys 1995;31:1341-6.

8. National Cancer Institute. Common Terminology Criteria for Adverse Events (CTCAE) v5.0 [Internet]. Bethesda, MD: National Cancer Institute; 2017 [cited 2020 Dec 2]. Available from: https://ctep.cancer.gov/protocoldevelopment/electronic_applications/ctc.htm\#ctc_50.

9. Langberg M, Rotem C, Fenig E, Koren R, Ravid A. Vitamin D protects keratinocytes from deleterious effects of ionizing radiation. Br J Dermatol 2009;160:151-61.

10. Richardson J, Smith JE, Mclntyre M, Thomas R, Pilkington K. Aloe vera for preventing radiation-induced skin reactions: a systematic literature review. Clin Oncol (R Coll Radiol) 2005;17:478-84.

11. Sharp L, Finnila K, Johansson H, Abrahamsson M, Hatschek T, Bergenmar M. No differences between Calendula cream and aqueous cream in the prevention of acute radiation skin reactions: results from a randomised blinded trial. Eur J Oncol Nurs 2013; 17:429-35.

12. Zhang $Y$, Zhang $S$, Shao $X$. Topical agent therapy for prevention and treatment of radiodermatitis: a meta-analysis. Support Care Cancer 2013;21:1025-31.

13. Nasser NJ, Fenig S, Ravid A, et al. Vitamin D ointment for prevention of radiation dermatitis in breast cancer patients. NPJ Breast Cancer 2017;3:10.

14. Pommier P, Gomez F, Sunyach MP, D'Hombres A, Carrie C, Montbarbon X. Phase III randomized trial of Calendula officinalis compared with trolamine for the prevention of acute dermatitis during irradiation for breast cancer. J Clin Oncol 2004;22:144753.

15. Bostrom A, Lindman $H$, Swartling $C$, Berne B, Bergh J. Potent corticosteroid cream (mometasone furoate) significantly reduces acute radiation dermatitis: results from a double-blind, randomized study. Radiother Oncol 2001;59:257-65.

16. Hindley A, Zain $Z$, Wood $L$, et al. Mometasone furoate cream reduces acute radiation dermatitis in patients receiving breast radiation therapy: results of a randomized trial. Int J Radiat Oncol Biol Phys 2014;90:748-55.

17. Pignol JP, Olivotto I, Rakovitch $E_{1}$ et al. A multicenter randomized trial of breast intensity-modulated radiation therapy to reduce acute radiation dermatitis. J Clin Oncol 2008;26:2085-92.

18. Diggelmann KV, Zytkovicz AE, Tuaine JM, Bennett NC, Kelly LE, Herst PM. Mepilex Lite dressings for the management of radiation-induced erythema: a systematic inpatient controlled clinical trial. Br J Radiol. 2010;83:971-8.

19. Zhao H, Zhu W, Jia L, et al. Phase I study of topical epigallocatechin-3-gallate (EGCG) in patients with breast cancer receiving adjuvant radiotherapy. Br J Radiol 2016;89:20150665.

20. Yan J, Yuan L, Wang J, et al. Mepitel Film is superior to Biafine cream in managing acute radiation-induced skin reactions in head and neck cancer patients: a randomised intra-patient controlled clinical trial. J Med Radiat Sci 2020;67:208-16. 